\title{
On the Existence and Uniqueness for High Order Fuzzy Fractional Differential Equations with Uncertainty
}

\author{
Abdourazek Souahi, ${ }^{1}$ Assia Guezane-Lakoud, ${ }^{2}$ and Amara Hitta ${ }^{1}$ \\ ${ }^{1}$ Laboratory of Applied Mathematics and Modeling, University of 8 May 1945 Guelma, P.O. Box 401, 24000 Guelma, Algeria \\ ${ }^{2}$ Laboratory of Advanced Materials, University of Badji Mokhtar-Annaba, P.O. Box 12, 23000 Annaba, Algeria
}

Correspondence should be addressed to Abdourazek Souahi; arsouahi@yahoo.fr

Received 30 November 2015; Revised 12 April 2016; Accepted 21 April 2016

Academic Editor: Ning Xiong

Copyright (c) 2016 Abdourazek Souahi et al. This is an open access article distributed under the Creative Commons Attribution License, which permits unrestricted use, distribution, and reproduction in any medium, provided the original work is properly cited.

A class fuzzy fractional differential equation (FFDE) involving Riemann-Liouville $H$-differentiability of arbitrary order $q>1$ is considered. Using Krasnoselskii-Krein type conditions, Kooi type conditions, and Rogers conditions we establish the uniqueness and existence of the solution after determining the equivalent integral form of the solution.

\section{Introduction}

In the last several years, fractional differential equations attracted more and more researchers and have proven to be very useful tools for modeling phenomena in physics, finance, and many other areas. The Riemann-Liouville formulation arises in a natural way for problems such as transport problems from the continuum random walk scheme or generalizes Chapman-Kolmogorov models $[1,2]$. It was also applied for modeling the behavior of viscoelastic and viscoplastic materials under external influences $[3,4]$ and the continuum and statistical mechanics for viscoelasticity problems [5]. Some of the several research papers were published to consider the uniqueness of the solution for fractional differential equations under Nagumo like conditions (see, e.g., [6-13] and references therein). In [11, 12], Lakshmikantham and Leela established the uniqueness of the solution for the problem $D^{q} x(t)=f(t, x(t))$, where $0<q<1$. Then, in [13], Yoruk et al. proved the uniqueness of the solution via KrasnoselskiiKrein, Rogers, and Kooi conditions, for $1<q<2$.

On the other hand, in order to obtain more realistic modeling of phenomena, one has to take uncertainty; see [1416] and the references cited therein. Many researchers have worked in the theoretical and numerical aspect of fractional and fuzzy differential equations; the reader is kindly referred to $[2,17-31]$ and the references therein.
In [32], Allahviranloo and Ahmadi introduced the fuzzy Laplace transform, which they used under the strongly generalized differentiability. Recently, ElJaoui et al. [33] developed it further. The newly defined fuzzy Laplace transform [32] for high order fuzzy derivatives is one of the most useful methods as mentioned by Jafarian et al. in [34]: “..., one of the important and interesting transforms in the problems of fuzzy equations is Laplace transforms. The fuzzy Laplace transform method solves fuzzy fractional differential equations and fuzzy boundary and initial value problems [35-38] ...."

Motivated by the above works, we adopted the fuzzy Laplace transform to prove the uniqueness and existence for the following initial value problems (FFDE) for arbitrary order $q>1$ :

$$
\begin{aligned}
D^{q} x(t) & =f\left(t, x(t), D^{q-1} x(t)\right), \\
x(0) & =y_{0}, \\
D^{(q-i)} x(0) & =\widetilde{0}, \quad i=1, \ldots,[q],
\end{aligned}
$$

where $y_{0} \in \mathbb{E}$ and $f: \mathbb{E}_{0} \rightarrow \mathbb{E}$ is a continuous fuzzy-valued function with

$$
\mathbb{E}_{0}=\{(t, x): 0 \leq t \leq 1, d(x(t), \widetilde{0}) \leq b\},
$$

where $d$ is the Hausdorff distance. 
Our aim is to both generalize and extend the previous uniqueness results of $[13,39]$.

The organization of this paper is as follows. Section 2 contains some basic definitions concerning fuzzy set theory and Riemann-Liouville generalized $H$-differentiability. In Section 3, using the fuzzy Laplace transform we determine the equivalent integral problem. Section 4 is devoted to the main results: a Krasnoselskii-Krein type of uniqueness theorem, a Kooi type uniqueness theorem, and a Rogers type uniqueness theorem; then we prove that the successive approximations converge to the unique solution.

\section{Preliminaries}

First, let us recall some basic definitions about fuzzy numbers and fuzzy sets. Here and in the rest of the paper, we denote by $\Gamma$ the Gamma function and $[\alpha]$ the integer part of $\alpha$.

As defined in $[40], \mathbb{E}=\{u: \mathbb{R} \rightarrow[0,1] ; u$ satisfies (A1)-(A4) $\}$ is the space of fuzzy numbers:

(A1) $u$ is normal; that is, there exists $y_{0} \in \mathbb{R}$ such that $u\left(y_{0}\right)=1$.

(A2) $u$ is fuzzy convex; that is, $u(\lambda y+(1-\lambda) z) \geq$ $\min \{u(y), u(z)\}$ whenever $y, z \in \mathbb{R}$ and $\lambda \in[0,1]$.

(A3) $u$ is upper semicontinuous; that is, for any $y_{0} \in \mathbb{R}$ and $\varepsilon>0$ there exists $\delta\left(y_{0}, \varepsilon\right)>0$ such that $u(y)<$ $u\left(y_{0}\right)+\varepsilon$ whenever $\left|y-y_{0}\right|<\delta, y \in \mathbb{R}^{n}$.

(A4) The closure of the set $\{y \in \mathbb{R} ; u(y)>0\}$ is compact.

The set $[u]^{\alpha}=\{u \in \mathbb{R} ; u(y) \geq \alpha\}$ is called $\alpha$-level set of $u$.

It follows from (A1)-(A4) that the $\alpha$-level sets $[u]^{\alpha}$ are convex compact subsets of $\mathbb{R}$ for all $\alpha \in(0,1]$. The fuzzy zero is defined by

$$
\widetilde{0}= \begin{cases}0 & \text { if } y \neq 0, \\ 1 & \text { if } y=0\end{cases}
$$

Definition 1 (see [40]). A fuzzy number $u$ in the parametric form is a pair $(\underline{u}(r), \bar{u}(r))$ of functions $\underline{u}(r), \bar{u}(r), 0 \leq r \leq 1$, which satisfy the following conditions:

(1) $\underline{u}(r)$ is a bounded nondecreasing left continuous function in $(0,1]$ and right continuous at 0 ;

(2) $\bar{u}(r)$ is a bounded nonincreasing left continuous function in $(0,1]$ and right continuous at 0 ;

(3) $\underline{u}(r) \leq \bar{u}(r), 0 \leq r \leq 1$.

Moreover, we also can present the $r$-cut representation of fuzzy numbers as $[u]^{r}=[\underline{u}(r), \bar{u}(r)]$ for all $0 \leq r \leq 1$.

According to Zadeh's extension principle, we have the following properties of fuzzy addition and multiplication by scalar on $\mathbb{E}$ :

$$
\begin{aligned}
& (u \oplus v)(x)=\sup _{y \in \mathbb{R}} \min \{u(y), v(x-y)\}, \quad x \in \mathbb{R}, \\
& (k \odot u(x))= \begin{cases}u\left(\frac{x}{k}\right) & \text { if } k \geq 0, \\
\tilde{0} & \text { if } k=0 .\end{cases}
\end{aligned}
$$

Seeking simplicity, we note $\oplus, \odot$ by the usual,$+ \cdot$. The Hausdorff distance between the fuzzy numbers is denoted by $d$ : $\mathbb{E} \times \mathbb{E} \rightarrow\left[0,+\infty\left[\right.\right.$, such that $d(u, v)=\sup _{r \in[0,1]} \max \{\mid \underline{u}(r)-$ $\underline{v}(r)|,| \bar{u}(r)-\bar{v}(r) \mid\}$. And $(d, \mathbb{E})$ is a complete metric space.

Definition 2. Let $x, y \in \mathbb{E}$. If there exists $z \in \mathbb{E}$ such that $x=y+z$, then $z$ is called the $H$-difference of $x$ and $y$, and it is denoted by $x \ominus y$.

Remark 3. Note that the sign $\ominus$ stands for $H$-difference and $x \ominus y \neq x+(-1) y$.

We denote by $C^{\mathbb{F}}[0, a]$ the space of all fuzzy-valued functions which are continuous on $[0, a]$ and $L^{\mathbb{F}}[0, a]$ the space of all Lebesgue integrable fuzzy-valued functions on $[0, a]$, where $a>0$. We also denote by $A C^{(n-1) \mathbb{F}}[0, a]$ the space of fuzzy-valued functions $f$ which have continuous $H$ derivatives up to order $n-1$ on $[0, a]$ such that $f^{(n-1)}$ in $A C^{\mathbb{F}}[0, a]$.

Definition 4 (see [41]). Let $f \in C^{\mathbb{F}}[0,1] \cap L^{\mathbb{F}}[0,1]$. The fuzzy fractional integral of the fuzzy-valued function $f$ is defined by

$$
I^{\beta} f(x ; r)=\left[I^{\beta} \underline{f}(x ; r), I^{\beta} \bar{f}(x ; r)\right], \quad 0 \leq r \leq 1,
$$

where

$$
\begin{aligned}
& I^{\beta} \underline{f}(x ; r)=\frac{1}{\Gamma(\beta)} \int_{0}^{x}(x-s)^{\beta-1} \underline{f}(s ; r) d s, \\
& I^{\beta \bar{f}}(x ; r)=\frac{1}{\Gamma(\beta)} \int_{0}^{x}(x-s)^{\beta-1} \bar{f}(s ; r) d s .
\end{aligned}
$$

Definition 5 (see [41, Definition 6]). Let $f \in C^{(n) \mathbb{F}}[0,1] \cap$ $L^{\mathbb{F}}[0,1], x_{0} \in(0,1)$, and $\Phi(x)=(1 / \Gamma(n-\beta)) \int_{0}^{x}(f(t) d t /(x-$ $\left.t)^{\beta-n+1}\right)$, where $n=[\beta]+1$. One says that $f$ is fuzzy RiemannLiouville fractional differentiable of order $\beta$ at $x_{0}$, if there exists an element $\left(D_{0}^{\beta} f\right)\left(x_{0}\right) \in \mathbb{E}$, such that, for all $h>0$ sufficiently small, one has

(i)

$$
\begin{aligned}
\left(D_{0}^{\beta} f\right)\left(x_{0}\right) & =\lim _{h \rightarrow 0} \frac{\Phi^{(n-1)}\left(x_{0}+h\right) \ominus \Phi^{(n-1)}\left(x_{0}\right)}{h} \\
& =\lim _{h \rightarrow 0} \frac{\Phi^{(n-1)}\left(x_{0}\right) \ominus \Phi^{(n-1)}\left(x_{0}-h\right)}{h}
\end{aligned}
$$

or

(ii)

$$
\begin{aligned}
\left(D_{0}^{\beta} f\right)\left(x_{0}\right) & =\lim _{h \rightarrow 0} \frac{\Phi^{(n-1)}\left(x_{0}\right) \ominus \Phi^{(n-1)}\left(x_{0}+h\right)}{h} \\
& =\lim _{h \rightarrow 0} \frac{\Phi^{(n-1)}\left(x_{0}-h\right) \ominus \Phi^{(n-1)}\left(x_{0}\right)}{h} .
\end{aligned}
$$


Denote by $C^{(n-1) \mathbb{F}}([0, a])$ the space of fuzzy-valued functions $f$ on the bounded interval $[0, a]$ which have continuous $H$-derivative up to order $n-2$ such that $f^{(n-1)} \in C^{\mathbb{F}}[0, a]$. $C^{(n-1) \mathbb{F}}([0, a])$ is a complete metric space endowed by the metric $D$ such that for every $g, h \in C^{(n-1) \mathbb{F}}([0, a])$

$$
D(g, h)=\sum_{i=0}^{n-1} \sup _{t \in[0, a]} d\left(g^{(i)}(t), h^{(i)}(t)\right) .
$$

In the rest of the paper, we say that a fuzzy-valued function $f$ is ${ }^{R L}[(i)-\beta]$-differentiable if it is differentiable as in Definition 5 case (i) and is ${ }^{R L}[(i i)-\beta]$-differentiable if it is differentiable as in Definition 5 case (ii).

Definition 6 (see [41, Theorem 7]). Let $f \in C^{(n) \mathbb{F}}[0,1] \cap$ $L^{\mathbb{F}}[0,1], x_{0} \in(0,1)$, and $\Phi(x)=(1 / \Gamma(n-\beta)) \int_{0}^{x}(f(t) d t /(x-$ $\left.t)^{\beta-n+1}\right)$, where $n=[\beta]+1$ such that $0 \leq r \leq 1$; then one has the following:

(i) if $f$ is ${ }^{R L}[(i)-\beta]$-differentiable fuzzy-valued function, then

$$
\left(D_{0}^{\beta} f\right)\left(x_{0} ; r\right)=\left[\left(D_{0}^{\beta} \underline{f}\right)\left(x_{0} ; r\right),\left(D_{0}^{\beta} \bar{f}\right)\left(x_{0} ; r\right)\right] \text {, }
$$

or

(ii) if $f$ is ${ }^{R L}[(i)-\beta]$-differentiable fuzzy-valued function, then

$$
\left(D_{0}^{\beta} f\right)\left(x_{0} ; r\right)=\left[\left(D_{0}^{\beta} \bar{f}\right)\left(x_{0} ; r\right),\left(D_{0}^{\beta} \underline{f}\right)\left(x_{0} ; r\right)\right] \text {, }
$$

where

$$
\begin{gathered}
\left(D_{0}^{\beta} \underline{f}\right)\left(x_{0} ; r\right)=\left[\frac{1}{\Gamma(n-\beta)}\left(\frac{d}{d x}\right)^{n}\right. \\
\left.\cdot \int_{0}^{x}(x-t)^{n-\beta-1} \underline{f}(t ; r) d t\right]_{x=x_{0}}, \\
\left(D_{0}^{\beta \bar{f}}\right)\left(x_{0} ; r\right)=\left[\frac{1}{\Gamma(n-\beta)}\left(\frac{d}{d x}\right)^{n}\right. \\
\left.\cdot \int_{0}^{x}(x-t)^{n-\beta-1} \bar{f}(t ; r) d t\right]_{x=x_{0}} .
\end{gathered}
$$

The following theorem is an important one about the fuzzy Laplace transform $\mathbf{L}$ of the Riemann-Liouville $H$ derivative for fuzzy-valued functions.

Theorem 7. Suppose that $f \in C^{(n) \mathbb{F}}[0, \infty) \cap L^{\mathbb{E}}[0, \infty)$; one has the following:

(i) if $f$ is ${ }^{R L}[(i)-\beta]$-differentiable fuzzy-valued function, then

$\mathbf{L}\left[\left(D_{0}^{\beta} f\right)(x)\right]=p^{\beta} \mathbf{L}[f(t)] \ominus\left(\sum_{k=0}^{n-1} p^{k} D^{\beta-k-1} f\right)(0)$ (ii) if $f$ is ${ }^{R L}[(i)-\beta]$-differentiable fuzzy-valued function, then

$$
\begin{aligned}
\mathbf{L}\left[\left(D_{0}^{\beta} f\right)(x)\right]= & -\left(\sum_{k=0}^{n-1} p^{k} D^{\beta-k-1} f\right)(0) \\
& \ominus\left(-p^{\beta} \mathbf{L}[f(t)]\right) .
\end{aligned}
$$

The proof runs along similar lines as that of [41, Theorem 16], and we omit it here.

\section{Fuzzy Fractional Integral Equation}

In this section, we study the relation between problem (1) and the fuzzy integral form using the well-known fuzzy Laplace transform.

In fact, by taking Laplace transform on both sides of

$$
D^{q} x(t)=f\left(t, x(t), D^{q-1} x(t)\right) \triangleq r(t, x)
$$

we get

$$
\mathbf{L}\left[D^{q} x(t)\right]=\mathbf{L}\left[f\left(t, x(t), D^{q-1} x(t)\right)\right] .
$$

Based on the type of Riemann-Liouville $H$-differentiability, we obtain two cases.

Case 1. If $D^{q} x$ is ${ }^{R L}[(i)-q]$-differentiable fuzzy-valued function, then

$$
\mathbf{L} r(t, x)=-\left(\sum_{k=0}^{n-1} p^{k} D^{\beta-k-1} x\right)(0) \ominus p^{q} \mathbf{L}[x(t)],
$$

and based on the lower and upper functions of $D^{q} x$ the above equation becomes

$$
\begin{aligned}
& \mathbf{L}[\underline{r}(t, x ; r)]=p^{q} \mathbf{L}[\underline{x}(t ; r)]-\sum_{k=0}^{n-1} p^{k} D^{\beta-k-1} \underline{x}(0 ; r), \\
& \mathbf{L}[\bar{r}(t, x ; r)]=p^{q} \mathbf{L}[\bar{x}(t ; r)]-\sum_{k=0}^{n-1} p^{k} D^{\beta-k-1} \bar{x}(0 ; r),
\end{aligned}
$$

where

$$
\begin{array}{r}
\mathbf{L}[\underline{r}(t, x ; r)]=\min \{r(t, u) \mid u \in[\underline{x}(t ; r), \bar{x}(t ; r)]\}, \\
0 \leq r \leq 1, \\
\mathbf{L}[\bar{r}(t, x ; r)]=\max \{r(t, u) \mid u \in[\underline{x}(t ; r), \bar{x}(t ; r)]\}, \\
0 \leq r \leq 1 .
\end{array}
$$

In order to solve system (18), and for the sake of simplicity, we assume that

$$
\begin{aligned}
& \mathbf{L}[\underline{x}(t ; r)]=H_{1}(p ; r), \\
& \mathbf{L}[\bar{x}(t ; r)]=K_{1}(p ; r),
\end{aligned}
$$


where $H_{1}(p ; r)$ and $K_{1}(p ; r)$ are solutions of the previous system (18); it yields

$$
\begin{aligned}
& \underline{x}(t ; r)=\mathbf{L}^{-1}\left[H_{1}(p ; r)\right], \\
& \bar{x}(t ; r)=\mathbf{L}^{-1}\left[K_{1}(p ; r)\right] .
\end{aligned}
$$

Case 2. If $D^{q} x$ is ${ }^{R L}[(i i)-q]$-differentiable fuzzy-valued function, then

$$
\mathbf{L} r(t, x)=p^{q} \mathbf{L}[x(t)] \ominus\left(\sum_{k=0}^{n-1} p^{k} D^{\beta-k-1} x\right)(0),
$$

and based on the lower and upper functions of $D^{q} x$ the above equation becomes

$$
\begin{aligned}
& \mathbf{L}[\underline{r}(t, x ; r)]=p^{q} \mathbf{L}[\underline{x}(t ; r)]-\sum_{k=0}^{n-1} p^{k} D^{\beta-k-1} \underline{x}(0 ; r), \\
& \mathbf{L}[\bar{r}(t, x ; r)]=p^{q} \mathbf{L}[\bar{x}(t ; r)]-\sum_{k=0}^{n-1} p^{k} D^{\beta-k-1} \bar{x}(0 ; r),
\end{aligned}
$$

where

$$
\begin{array}{r}
\mathbf{L}[\underline{r}(t, x ; r)]=\min \{r(t, u) \mid u \in[\underline{x}(t ; r), \bar{x}(t ; r)]\}, \\
0 \leq r \leq 1, \\
\mathbf{L}[\bar{r}(t, x ; r)]=\max \{r(t, u) \mid u \in[\underline{x}(t ; r), \bar{x}(t ; r)]\}, \\
0 \leq r \leq 1 .
\end{array}
$$

In order to solve system (23), and for the sake of simplicity, we assume that

$$
\begin{aligned}
& \mathbf{L}[\underline{x}(t ; r)]=H_{2}(p ; r), \\
& \mathbf{L}[\bar{x}(t ; r)]=K_{2}(p ; r),
\end{aligned}
$$

where $H_{2}(p ; r)$ and $K_{2}(p ; r)$ are solutions of the previous system (23). Then we obtain

$$
\begin{aligned}
& \underline{x}(t ; r)=\mathbf{L}^{-1}\left[H_{2}(p ; r)\right], \\
& \bar{x}(t ; r)=\mathbf{L}^{-1}\left[K_{2}(p ; r)\right] .
\end{aligned}
$$

Taking into account the initial conditions of problem (1) and using the linearity of the inverse Laplace transform on systems (20) and (26), we obtain the following for both cases.

$x$ is a solution for problem (1) if and only if $x$ is a solution for the following integral equation:

$$
x(t)=y_{0}+\frac{1}{\Gamma(q)} \int_{0}^{t}(t-s)^{q-1} r(s, x) d s
$$

in the sense of ${ }^{R L}[(i)-q]$-differentiability, and

$$
\widehat{x}(t)=y_{0} \ominus \frac{-1}{\Gamma(q)} \int_{0}^{t}(t-s)^{q-1} r(s, x) d s
$$

in the sense of ${ }^{R L}[(i i)-q]$-differentiability.

\section{Main Results}

Now, we state the Krasnoselskii-Krein type conditions for FFDE (1).

Theorem 8. Let $f \in C\left(\mathbb{E}_{0}, \mathbb{E}\right)$ satisfy the following Krein type conditions:

(H1) $d(f(t, x, y), f(t, \bar{x}, \bar{y})) \leq \min \{\Gamma(q), 1\}((k+\alpha(q-$ $\left.[q])) / 2 t^{1-\alpha(q-[q])}\right)[d(x, \bar{x})+d(y, \bar{y})], t \neq 0$ and $0<$ $\alpha<1$,

$(\mathrm{H} 2) d(f(t, x, y), f(t, \bar{x}, \bar{y})) \leq \delta d(x, \bar{x})^{\alpha}+t^{\alpha(q-[q])} d(y, \bar{y})^{\alpha}$,

where $\delta$ and $k$ are positive constants and $k(1-\alpha)<1+$ $\alpha(q-[q])$; then in the sense of ${ }^{R L}[(i)-q]$-differentiability, the solution $x$ is unique and in the sense of ${ }^{R L}[(i)-q]$-differentiability, the solution $\hat{x}$ is unique on $[0, \eta]$, where $\eta=\min \{1$, $\left.(b \Gamma(1+q) / M)^{1 / q}, d / M\right\}$ and $M$ is the bound for $f$ on $E_{0}$ : that is, $d(f, \widetilde{0}) \leq M$.

Proof. First we establish the uniqueness; suppose $x$ and $y$ are any two solutions of (1) in ${ }^{R L}[(i)-q]$-differentiability and let $\phi(t)=d(x(t), y(t))$ and $\theta(t)=d\left(D^{q-1} x(t), D^{q-1} y(t)\right)$. Note that $\phi(0)=\theta(0)=0$.

We define $R(t)=\int_{0}^{t}\left[\phi^{\alpha}(s)+s^{\alpha(q-[q])} \theta^{\alpha}(s)\right] d s$; clearly $R(0)=0$.

Using (27) and condition (H2), we get

$$
\begin{aligned}
\phi(t) & \leq \frac{\delta}{\Gamma(q)} \int_{0}^{t}(t-s)^{q-1}\left[\phi^{\alpha}(s)+s^{\alpha(q-[q])} \theta^{\alpha}(s)\right] d s \\
& \leq \frac{\delta}{\Gamma(q)} t^{q-1} R(t), \\
\theta(t) & \leq \int_{0}^{t} \delta \phi(s)^{\alpha}+t^{\alpha(q-[q])} \theta(s)^{\alpha} d s \leq \delta R(t) .
\end{aligned}
$$

For the sake of simplicity we use the same symbol $C$ to denote all different constants arising in the rest of the proof.

We have

$$
\begin{aligned}
R^{\prime}(t) & =\phi^{\alpha}(t)+t^{\alpha(q-[q])} \theta^{\alpha}(t) \\
& \leq C\left[t^{\alpha(q-1)}+t^{\alpha(q-[q])}\right] R^{\alpha}(t) .
\end{aligned}
$$

Since $R(t)>0$ for $t>0$, multiplying both sides of (30) by $(1-\alpha) R^{-\alpha}(t)$ and then integrating the resulting inequality, we get

$$
R(t) \leq C\left(t^{((\alpha /(1-\alpha)) q+1)}+t^{((\alpha /(1-\alpha)) q+(1-\alpha[q]) /(1-\alpha))}\right) .
$$

Using the fact that

$$
(a+b)^{(1-\alpha)} \leq \frac{1}{2^{(1-\alpha)-1}}\left(a^{(1-\alpha)}+b^{(1-\alpha)}\right)
$$

for every $a, b \in(0,1)$, (31) becomes

$$
R(t) \leq C\left(t^{((\alpha /(1-\alpha)) q+1)}+t^{((\alpha /(1-\alpha)) q+(1-\alpha[q]) /(1-\alpha))}\right) .
$$


This leads to the following estimates on $\phi$ and $\theta$, for $t \in[0, \eta]$ :

$$
\begin{aligned}
& \phi(t) \leq C\left(t^{(q /(1-\alpha))}+t^{(q /(1-\alpha)+\alpha(1-[q]) /(1-\alpha))}\right), \\
& \theta(t) \leq C\left(t^{((\alpha /(1-\alpha)) q+1)}+t^{((\alpha /(1-\alpha)) q+(1-\alpha[q]) /(1-\alpha))}\right) .
\end{aligned}
$$

Define the function $\psi(t)=t^{-k} \max \{\phi(t), \theta(t)\}$ for $t \in(0,1]$. When either $t^{-k} \phi(t)$ or $t^{-k} \theta(t)$ is the maximum, we get

$$
0 \leq \psi(t) \leq C\left(t^{(q /(1-\alpha)-k)}+t^{(q /(1-\alpha)+\alpha(1-[q]) /(1-\alpha)-k)}\right),
$$

or

$$
\begin{aligned}
0 & \leq \psi(t) \\
& \leq C\left(t^{((\alpha /(1-\alpha)) q+1-k)}+t^{(\alpha q /(1-\alpha)+(1-\alpha[q]) /(1-\alpha)-k)}\right) .
\end{aligned}
$$

Since $k(1-\alpha)<1+\alpha(q-[q])$ (by assumption), we have

$$
\begin{aligned}
k(1-\alpha) & <1+\alpha(q-[q]) \Longrightarrow \\
k(1-\alpha) & <q \\
(k-1)(1-\alpha) & <\alpha q \\
k(1-\alpha) & <q+\alpha-\alpha[q] \\
k(1-\alpha) & <\alpha q+1-\alpha[q] .
\end{aligned}
$$

So all of the exponents of $t$ in the above inequalities are positive. Hence, $\lim _{t \rightarrow 0^{+}} \psi(t)=0$. Therefore, if we define $\psi(0)=0$, the function $\psi$ is continuous in $[0, \eta]$.

We want to prove that $\psi \equiv 0$. In fact, since the function $\psi$ is continuous, if $\psi$ does not vanish at some points $t$, that is, $\psi(t)>0$ on $] 0, \eta]$, then there exists a maximum $m>0$ reached when $t$ is equal to some $t_{1}: 0<t_{1} \leq \eta \leq 1$ such that $\psi(s)<m=\psi\left(t_{1}\right)$, for $\left.\left.s \in\right] 0, t_{1}\right)$. But, from condition (H1) we get for either cases

$$
\begin{aligned}
m & =\psi\left(t_{1}\right)=t_{1}^{-k} \phi\left(t_{1}\right) \leq \min (\Gamma(q), 1) m t_{1}^{q-1+\alpha(q-[q])} \\
& <m
\end{aligned}
$$

or

$$
\begin{aligned}
m & =\psi\left(t_{1}\right)=t_{1}^{-k} \theta\left(t_{1}\right) \leq \min (\Gamma(q), 1) m t_{1}^{\alpha(q-[q])} \\
& <m
\end{aligned}
$$

which is a contradiction. Thus, the uniqueness of the solution is established in the sense of ${ }^{R L}[(i)-q]$-differentiability. The second part of the proof is almost completely similar to the $R L[(i)-q]$-differentiability; thus, we omit it.

Remark 9. For the case $1<q<2$, of the deterministic case, Theorem 8 is reduced to [13, Theorem 3.1].

Theorem 10 (Kooi's type uniqueness theorem). Let $f$ satisfy the following conditions:

(J1) $d(f(t, x, y), f(t, \bar{x}, \bar{y})) \leq \min \{\Gamma(q), 1\}((k+\alpha(q-[q])) /$ $\left.2 t^{1-\alpha(q-[q])}\right)[d(x, \bar{x})+d(y, \bar{y})], t \neq 0$ and $0<\alpha<1$;
(J2) $t^{\beta} d(f(t, x, y), f(t, \bar{x}, \bar{y})) \leq c\left[d(x, \bar{x})^{\alpha}+t^{\alpha(q-[q])} d(y\right.$, $\left.\bar{y})^{\alpha}\right]$

where $c$ and $k$ are positive constants and $k(1-\alpha)<1+$ $\alpha(q-[q])-\beta$, for $(t, x, y),(t, \bar{x}, \bar{y}) \in R_{0}$; then in the sense of ${ }^{R L}[(i)-q]$-differentiability, the solution $x$ is unique and in the sense of ${ }^{R L}[(i)-q]$-differentiability, the solution $\hat{x}$ is unique.

Proof. It is similar to that of Theorem 8; thus, we omit it.

Lemma 11. Let $\phi$ and $\theta$ be two nonnegative continuous functions in the interval $[0, \eta]$ for a real number $a>0$. Let $\psi(t)=\int_{0}^{t}\left(\left(\phi(s)+s^{q-[q]} \theta(s)\right) / 2 s^{q-[q]+2}\right) d s$. Assume the following:

(i) $\phi(t) \leq t^{q-[q]} \psi(t)$,

(ii) $\theta(t) \leq \psi(t)$,

(iii) $\phi(t)=o\left(t^{q-[q]} e^{-1 / t}\right)$,

(iv) $\theta(t)=o\left(e^{-1 / t}\right)$.

Then $\phi \equiv \theta \equiv 0$.

Proof. Let $\psi(t)=\int_{0}^{t}\left(\left(\phi(s)+s^{q-[q]} \theta(s)\right) / 2 s^{q-[q]+2}\right) d s$. After differentiating $\psi$ and using (ii), we obtain, for $t>0, \psi^{\prime}(t) \leq$ $\left(1 / t^{2}\right) \psi(t)$, so that $e^{1 / t} \psi(t)$ is decreasing. Now, from (iii) and (iv), if $\epsilon>0$ then, for a small $t$, we have

$$
e^{1 / t} \psi(t) \leq e^{1 / t} \int_{0}^{t} \frac{1}{2 s^{2}} 2 \epsilon e^{-1 / s} d s=\epsilon
$$

Hence, $\lim _{t \rightarrow 0} e^{1 / t} \psi(t)=0$ which implies that $\psi(t) \leq 0$. Finally, $\psi$ is nonnegative due to (i), and thus $\psi \equiv 0$.

Theorem 12 (Rogers' type uniqueness theorem). Let the function $f$ verify the following conditions:

(K1) $d(f(t, x, y), \widetilde{0}) \leq \min \{\Gamma(q), 1\} o\left(e^{-1 / t} / t^{2}\right)$, uniformly for positive and bounded $x$ and $y$ on $\mathbb{E}$,

(K2) $d(f(t, x, y), f(t, \bar{x}, \bar{y})) \leq \leq \quad \min \{\Gamma(q), 1\}(1 /$
$\left.2 t^{q-[q]+2}\right)\left[d(x, \bar{x})+t^{(q-[q])} d(y, \bar{y})\right]$.

Then the problem has at most one solution.

The proof of this theorem is essentially based on Lemma 11. 
Proof. Suppose $x$ and $y$ are any two solutions of (1) in $R L[(i)-q]$-differentiability, and let $\phi(t)=d(x(t), y(t))$ and $\theta(t)=d\left(D^{q-1} x(t), D^{q-1} y(t)\right)$; we get for $t \in[0, \eta] \subset[0,1]$

$$
\begin{aligned}
\phi(t) & \leq \frac{1}{\Gamma(q)} \int_{0}^{t}(t-s)^{q-1} d(r(s, x), r(s, y)) \\
& \leq \int_{0}^{t} \frac{(t-s)^{q-1}}{2 s^{q-[q]+2}}\left[\phi(s)+s^{q-[q]} \theta(s)\right] d s \\
& \leq t^{q-1} \int_{0}^{t} \frac{1}{2 s^{q-[q]+2}}\left[\phi(s)+s^{q-1} \theta(s)\right] d s \\
& \leq t^{q-[q]} \int_{0}^{t} \frac{1}{2 s^{q-[q]+2}}\left[\phi(s)+s^{q-1} \theta(s)\right] d s \\
& \leq t^{q-[q]} \psi(t), \\
\theta(s) & \leq \int_{0}^{t} d(r(s, x), r(s, y)) \\
& \leq \int_{0}^{t} \frac{\min \{\Gamma(q), 1\}}{2 s^{q-[q]+2}}\left[\phi(s)+s^{q-[q]} \theta(s)\right] d s \\
& \leq \int_{0}^{t} \frac{1}{2 s^{q-[q]+2}\left[\phi(s)+s^{q-[q]} \theta(s)\right] d s \leq \psi(t),}
\end{aligned}
$$

where $\psi$ is defined as in Lemma 11.

Also, if $\epsilon>0$, then from the condition (K1) for small $t$, we have

$$
\begin{aligned}
\phi(t) & \leq \frac{t^{q-1}}{\Gamma(q)} \int_{0}^{t} d(r(s, x), r(s, y)) \\
& <t^{q-1} 2 \epsilon \int_{0}^{t} \frac{e^{-1 / s}}{s^{2}} d s \leq t^{q-1} e^{-1 / t} 2 \epsilon \\
& <\epsilon t^{q-[q]} e^{-1 / t} 2 \\
\theta(t) & \leq \int_{0}^{t} d(r(s, x), r(s, y)) \\
& <2 \epsilon \min \{1, \Gamma(q)\} \int_{0}^{t} \frac{e^{-1 / s}}{s^{2}} d s \leq 2 \epsilon e^{-1 / t}
\end{aligned}
$$

By applying Lemma 11, we obtain $d(x(t), y(t))=0$ for every $t \in[0,1]$, and this proves the uniqueness of the solution of the FFDE (1) in ${ }^{R L}[(i)-q]$-differentiability. The second part of the proof is almost completely similar; thus, we omit it.

Theorem 13. Let $f \in C\left(\mathbb{E}_{0}, \mathbb{E}\right)$ satisfy the conditions of Theorem 8 . Then the successive approximations

$$
x_{n}(t)=y_{0}+\frac{1}{\Gamma(q)} \int_{0}^{t}(t-s)^{q-1} r\left(s, x_{n-1}\right) d s
$$

in the sense of ${ }^{R L}[(i)-q]$-differentiability or

$$
\widehat{x}_{n}(t)=y_{0} \ominus \frac{-1}{\Gamma(q)} \int_{0}^{t}(t-s)^{q-1} r\left(s, x_{n-1}\right) d s
$$

in the sense of ${ }^{R L}[(i i)-q]$-differentiability converge to the unique solution of the FFDE (1).
Proof. Without loss of generality, we prove Theorem 13 for the sequence $\left\{x_{n}\right\}$ in the sense of ${ }^{R L}[(i)-q]$-differentiability using Ascoli-Arzela Theorem. The convergence of the sequence $\left\{\hat{x}_{n}\right\}$ in the sense of ${ }^{R L}[(i i)-q]$-differentiability is completely similar so we omit it.

Step 1. The sequences $\left\{x_{j}\right\}_{j \geq 0}$ and $\left\{D^{q-1} x_{j}\right\}_{j \geq 0}$ are well defined and continuous and uniformly bounded on $[0, \eta]$; in fact

$$
\begin{aligned}
& d\left(x_{j+1}(t), y_{0}\right) \\
& \leq \frac{1}{\Gamma(q)} \int_{0}^{t}(t-s)^{q-1} d\left(r\left(s, x_{j}\right), \widetilde{0}\right) d s \\
& d\left(D^{q-1} x_{j+1}(t), y_{0}\right) \leq \int_{0}^{t} d\left(r\left(s, x_{j}\right), \tilde{0}\right) d s .
\end{aligned}
$$

For $j=0$ and $t \in[0, \eta]$, we have

$$
\begin{aligned}
d\left(x_{1}(t), y_{0}\right) & \leq \frac{M t^{q}}{\Gamma(q+1)} \leq b, \\
d\left(D^{q-1} x_{1}(t), y_{0}\right) & \leq M t \leq d .
\end{aligned}
$$

Moreover, for every $i \in\{0, \ldots, n-1\}$ we have

$$
\begin{aligned}
& d\left(x_{1}^{(i)}(t), \widetilde{0}\right)=d\left(D^{i} I^{q} f\left(t, x_{0}(t), D^{q-1} x_{0}(t)\right), \tilde{0}\right) \\
& =d\left(I^{q-i} f\left(t, x_{0}(t), D^{q-1} x_{0}(t)\right), \widetilde{0}\right)=\frac{1}{\Gamma(q-i)} \\
& \cdot \int_{0}^{t}(t-s)^{q-i-1} d\left(f\left(s, x_{0}(s), D^{q-1} x_{0}(s)\right), \widetilde{0}\right) d s \\
& \leq \frac{M}{\Gamma(q-i)} \int_{0}^{t}(t-s)^{q-i-1} d s \leq \frac{M t^{q-i}}{(q-i) \Gamma(q-i)} \\
& \leq \frac{M t^{q-i}}{\Gamma(q-i+1)} .
\end{aligned}
$$

By induction, the sequences $\left\{x_{j+1}(t)\right\}$ and $\left\{D^{q-1} x_{j+1}(t)\right\}$ are well defined and uniformly bounded on $[0, \eta]$.

Step 2. We prove that the functions $y$ and $z$ are continuous in $[0, \eta]$, where $y$ and $z$ are defined by

$$
\begin{aligned}
& y(t)=\limsup _{j \rightarrow \infty} \zeta_{j}^{0}(t), \\
& z(t)=\limsup _{j \rightarrow \infty} \xi_{j}(t),
\end{aligned}
$$

such that

$$
\begin{aligned}
& \zeta_{j}^{0}(t)=d\left(x_{j}(t), x_{j-1}(t)\right), \\
& \xi_{j}(t)=d\left(D^{q-1} x_{j}(t), D^{q-1} x_{j-1}(t)\right) .
\end{aligned}
$$

Let us note

$$
m(t)=\sum_{i \leq n-1} \limsup _{j \rightarrow \infty} \zeta_{j}^{i}(t)
$$


where

$$
\zeta_{j}^{i}(t)=d\left(x_{j}^{(i)}(t), x_{j-1}^{(i)}(t)\right) .
$$

For $0 \leq t_{1} \leq t_{2}$ and for every $i \in\{0, \ldots, n-1\}$, we obtain

$$
\begin{aligned}
& \left|\zeta_{j}^{i}\left(t_{1}\right)-\zeta_{j}^{i}\left(t_{2}\right)\right|=\mid d\left(x_{j+1}^{(i)}\left(t_{1}\right), x_{j}^{(i)}\left(t_{1}\right)\right) \\
& \quad-d\left(x_{j+1}^{(i)}\left(t_{2}\right), x_{j}^{(i)}\left(t_{2}\right)\right) \mid \\
& \quad \leq \frac{1}{\Gamma(q-i)}\left[\mid \int_{0}^{t_{1}}\left(t_{1}-s\right)^{q-1-i} d\left(r\left(s, x_{j}\right), r\left(s, x_{j-1}\right)\right) d s\right. \\
& \left.\quad-\int_{0}^{t_{2}}\left(t_{2}-s\right)^{q-1-i} d\left(r\left(s, x_{j}\right), r\left(s, x_{j-1}\right)\right) d s \mid\right] \\
& \quad \leq \frac{2 M}{\Gamma(q-i)}\left[\mid \int_{0}^{t_{1}}\left(\left(t_{1}-s\right)^{q-1-i}-\left(t_{2}-s\right)^{q-1-i}\right) d s\right. \\
& \left.\quad-\int_{t_{1}}^{t_{2}}\left(t_{2}-s\right)^{q-1-i} d s \mid\right] \leq \frac{2 M}{(q-i) \Gamma(q-i)}\left[t_{1}^{q-i}-t_{2}^{q-i}\right. \\
& \left.\quad+2\left(t_{2}-t_{1}\right)^{q-i}\right] \leq \frac{4 M}{\Gamma(q-i+1)}\left(t_{2}-t_{1}\right)^{q-i} .
\end{aligned}
$$

The right-hand side in the above inequalities is at most $(4 M / \Gamma(q-i+1))\left(t_{2}-t_{1}\right)^{q-i}+\epsilon$ for large $n$ if $\epsilon>0$ provided that

$$
\left|t_{2}-t_{1}\right| \leq \eta \leq \frac{4 M}{\Gamma(q-i+1)}\left(t_{2}-t_{1}\right)^{q-i},
$$

for every $i \leq n-1$. And since $\epsilon$ is arbitrary and $t_{1}, t_{2}$ can be interchangeable, we get

$$
\begin{aligned}
\left|m\left(t_{1}\right)-m\left(t_{2}\right)\right| & \leq \sum_{i \leq n-1}\left\{\frac{4 M}{\Gamma(q-i+1)}\left(t_{2}-t_{1}\right)^{q-i}\right\} \\
& \leq \frac{4 M(n-1)}{\Gamma(q+1)}\left(t_{2}-t_{1}\right)^{q} .
\end{aligned}
$$

The same goes for $z(t)$, and we obtain

$$
\left|z\left(t_{1}\right)-z\left(t_{2}\right)\right| \leq 2 M\left|t_{2}-t_{1}\right| \text {. }
$$

These imply that $y$ and $z$ are continuous on $[0, \eta]$.

Step 3 . We verify that the family $\left\{D^{q-1} j_{n+1}(t)\right\}$ is equicontinuous in $C^{\mathbb{F}}([0, \eta], \mathbb{E})$ and that the family $\left\{x_{j+1}(t)\right\}$ is equicontinuous in $C^{(n-1) \mathbb{F}}([0, \eta], \mathbb{E})$.

We may prove that by using condition $(\mathrm{H} 2)$ and the definition of successive approximations (43) we obtain

$$
\begin{aligned}
& \zeta_{j+1}^{0}(t) \leq C \int_{0}^{t}(t-s)^{q-1}\left[\zeta_{j}^{0}(s)^{\alpha}+s^{\alpha(q-[q])} \xi_{j}(s)^{\alpha}\right] d s, \\
& \zeta_{j+1}^{i}(t) \\
& \quad \leq C \int_{0}^{t}(t-s)^{q-i-1}\left[\zeta_{j}^{0}(s)^{\alpha}+s^{\alpha(q-[q])} \xi_{j}(s)^{\alpha}\right] d s .
\end{aligned}
$$

As a consequence, we obtain the following estimation:

$$
\begin{aligned}
& D\left(x_{j+1}, x_{j}\right) \leq \sum_{i \leq n-1} C \int_{0}^{1}(1-s)^{q-i-1} \\
& \cdot\left[\left|x_{j}(s)-x_{j-1}(s)\right|^{\alpha}\right. \\
& \left.\quad+s^{\alpha(q-[q])}\left|D^{q-1} x_{j}(s)-D^{q-1} x_{j-1}(s)\right|^{\alpha}\right] d s .
\end{aligned}
$$

By Arzela-Ascoli Theorem, there exists a subsequence of integers $\left\{j_{k}\right\}$, such that

$$
\begin{aligned}
& d\left(x_{j_{k}}(t), x_{j_{k-1}}(t)\right) \longrightarrow y(t) \\
& \text { as } j_{k} \longrightarrow \infty, \\
& d\left(D^{q-1} x_{j_{k}}(t), D^{q-1} x_{j_{k-1}}(t)\right) \longrightarrow y(t) \\
& \text { as } j_{k} \longrightarrow \infty .
\end{aligned}
$$

Let us note

$$
\begin{aligned}
m^{*}(t) & =\limsup _{k \rightarrow \infty} d\left(x_{j_{k}}(t)-x_{j_{k-1}}(t)\right), \\
z^{*}(t) & =\limsup _{k \rightarrow \infty} d\left(D^{q-1} x_{j_{k}}(t)-D^{q-1} x_{j_{k-1}}(t)\right) .
\end{aligned}
$$

Further, if $\left\{d\left(x_{j}, x_{j-1}\right)\right\} \rightarrow 0$ and $\left\{d\left(D^{q-1} x_{j}, D^{q-1} x_{j-1}\right)\right\} \rightarrow 0$ as $j \rightarrow \infty$, then the limit of any successive approximation of $x_{n}$ is the solution $x$ of (1), which was proved to be unique in Theorem 8. It follows that a selection of subsequences is unnecessary and that the entire sequence $\left\{x_{j}\right\}$ converges uniformly to $x(t)$. For that, it is sufficient to show that $y \equiv 0$ and $z \equiv 0$ which will lead to $m^{*}(t)$ and $z^{*}(t)$ being null.

Setting

$$
R(t)=\int_{0}^{t}\left[y(s)^{\alpha}+s^{\alpha(q-[q])} z(s)^{\alpha}\right] d s,
$$

and by defining $\psi^{*}(t)=t^{-k} \max \{y(t), z(t)\}$, we show that $\lim _{t \rightarrow 0^{+}} \psi^{*}(t)=0$.

Now we shall prove that $\psi^{*}(t) \equiv 0$. Suppose that $\psi^{*}(t)>0$ at any point in $[0, \eta]$; then there exists $t_{1}$ such that $0<\bar{m}=$ $\psi^{*}\left(t_{1}\right)=\max _{0 \leq t \leq \eta} \psi^{*}(t)$. Hence, from condition (H1), we obtain

$$
\begin{aligned}
\bar{m} & =\psi\left(t_{1}\right)=t_{1}^{-k} y\left(t_{1}\right) \leq \min (\Gamma(q), 1) \bar{m} t_{1}^{q-1+\alpha(q-[q])} \\
& <\bar{m}
\end{aligned}
$$

or

$$
\begin{aligned}
\bar{m} & =\psi\left(t_{1}\right)=t_{1}^{-k} z\left(t_{1}\right) \leq \min (\Gamma(q), 1) \bar{m} t_{1}^{\alpha(q-[q])} \\
& <\bar{m} .
\end{aligned}
$$

In both cases, we end up with a contradiction. So $\psi^{*}(t) \equiv 0$. Therefore, iteration (43) converges uniformly to the unique solution $x$ of $(1)$ on $[0, \eta]$. 


\section{Conclusion}

In this paper we established the uniqueness and existence of the solution under a fuzzy version of the Krasnoselskii-Krein conditions. Our work generalizes and extends the work of Yoruk et al. to arbitrary order in the fuzzy version. We finally hope to study other classes of fuzzy fractional differential problems in future works.

\section{Competing Interests}

The authors declare that there are no competing interests regarding the publication of this paper.

\section{References}

[1] R. Metzler, "Generalized Chapman-Kolmogorov equation: a unifying approach to the description of anomalous transport in external fields," Physical Review E, vol. 62, no. 5, part A, pp. 6233-6245, 2000.

[2] R. Metzler and J. Klafter, "The random walk's guide to anomalous diffusion: a fractional dynamics approach," Physics Reports, vol. 339, no. 1, 77 pages, 2000.

[3] K. Diethelm and A. D. Freed, "On the solution of nonlinear fractional-order differential equations used in the modeling of viscoplasticity," in Scientific Computing in Chenucal Engineering II: Computational Fluid Dynamics, Reaction Engineering, and Molecular Properties, F. Keil, W. Mackens, H. Vo13, and J. Werther, Eds., pp. 217-224, Springer, Heidelberg, Germany, 1999.

[4] A. A. Kilbas, H. M. Srivastava, and J. J. Trujillo, Theory and Applications of Fractional Differential Equations, vol. 204 of North-Holland Mathematics Studies, Elsevier Science B.V., Amsterdam, The Netherlands, 2006.

[5] F. Mainardi, "Fractional calculus: some basic problems in continuum and statistical mechanics," in Fractals and Fractional Calculus in Continuum Mechanics (Udine, 1996), vol. 378 of CISM Courses and Lectures, pp. 291-348, Springer, Vienna, Austria, 1997.

[6] R. P. Agarwal and V. Lakshmikantham, Uniqueness and Nonuniqueness Criteria for Ordinary Differential Equations, vol. 6 of Series in Real Analysis, World Scientific Publishing, River Edge, NJ, USA, 1993.

[7] A. Chidouh, A. Guezane-Lakoud, and R. Bebbouchi, "Positive solutions for an oscillator fractional initial value problem," Journal of Applied Mathematics and Computing, 2016.

[8] A. Chidouh, A. Guezane-Lakoud, and R. Bebbouchi, "Positive solutions of the fractional relaxation equation using lower and upper solutions," Vietnam Journal of Mathematics, 2016.

[9] K. Diethelm, N. J. Ford, and A. D. Freed, "A predictor-corrector approach for the numerical solution of fractional differential equations," Nonlinear Dynamics, vol. 29, no. 1-4, pp. 3-22, 2002.

[10] M. A. Krasnosel'skiı̌ and S. G. Kreinn, "On a class of uniqueness theorems for the equation $y^{\prime}=f(x, y)$," Uspekhi Matematicheskikh Nauk, vol. 11, no. 1(67), pp. 209-213, 1956.

[11] V. Lakshmikantham and S. Leela, "A Krasnoselskii-Krein-type uniqueness result for fractional differential equations," Nonlinear Analysis: Theory, Methods \& Applications, vol. 71, no. 78, pp. 3421-3424, 2009.

[12] V. Lakshmikantham and S. Leela, "Nagumo-type uniqueness result for fractional differential equations," Nonlinear Analysis:
Theory, Methods \& Applications, vol. 71, no. 7-8, pp. 2886-2889, 2009.

[13] F. Yoruk, T. G. Bhaskar, and R. P. Agarwal, "New uniqueness results for fractional differential equations," Applicable Analysis, vol. 92, no. 2, pp. 259-269, 2013.

[14] A. Ahmadian, S. Salahshour, D. Baleanu, H. Amirkhani, and R. Yunus, "Tau method for the numerical solution of a fuzzy fractional kinetic model and its application to the oil palm frond as a promising source of xylose," Journal of Computational Physics, vol. 294, pp. 562-584, 2015.

[15] Y. Chalco-Cano, A. Rufián-Lizana, H. Román-Flores, and M. D. Jiménez-Gamero, "Calculus for interval-valued functions using generalized Hukuhara derivative and applications," Fuzzy Sets and Systems, vol. 219, pp. 49-67, 2013.

[16] M. T. Malinowski, "Existence theorems for solutions to random fuzzy differential equations," Nonlinear Analysis: Theory, Methods \& Applications, vol. 73, no. 6, pp. 1515-1532, 2010.

[17] R. Alikhani, F. Bahrami, and A. Jabbari, "Existence of global solutions to nonlinear fuzzy Volterra integro-differential equations," Nonlinear Analysis: Theory, Methods \& Applications, vol. 75, no. 4, pp. 1810-1821, 2012.

[18] T. Allahviranloo, A. Armand, and Z. Gouyandeh, "Fuzzy fractional differential equations under generalized fuzzy Caputo derivative," Journal of Intelligent \& Fuzzy Systems, vol. 26, no. 3, pp. 1481-1490, 2014.

[19] T. Allahviranloo, S. Salahshour, and S. Abbasbandy, "Explicit solutions of fractional differential equations with uncertainty," Soft Computing, vol. 16, no. 2, pp. 297-302, 2012.

[20] T. V. An, N. D. Phu, and N. V. Hoa, "A note on solutions of interval-valued Volterra integral equations," Journal of Integral Equations and Applications, vol. 26, no. 1, pp. 1-14, 2014.

[21] M. Benchohra, J. J. Nieto, and A. Ouahab, "Fuzzy solutions for impulsive differential equations," Communications in Applied Analysis, vol. 11, no. 3-4, pp. 379-394, 2007.

[22] W. Fei, "Existence and uniqueness of solution for fuzzy random differential equations with non-Lipschitz coefficients," Information Sciences, vol. 177, no. 20, pp. 4329-4337, 2007.

[23] N. V. Hoa, "Fuzzy fractional functional differential equations under Caputo gH-differentiability," Communications in Nonlinear Science and Numerical Simulation, vol. 22, no. 1-3, pp. 11341157, 2015.

[24] N. V. Hoa, N. D. Phu, T. T. Tung, and L. T. Quang, "Intervalvalued functional integro-differential equations," Advances in Difference Equations, 2014:177, 20 pages, 2014.

[25] A. Khastan, F. Bahrami, and K. Ivaz, "New results on multiple solutions for $N$ th-order fuzzy differential equations under generalized differentiability," Boundary Value Problems, vol. 2009, Article ID 395714, 13 pages, 2009.

[26] Y. Li and J. Li, "Stability analysis of fractional order systems based on T-S fuzzy model with the fractional order $\alpha:<0<$ $\alpha<$ 1," Nonlinear Dynamics, vol. 78, no. 4, pp. 2909-2919, 2014.

[27] X. Liu, L. Zhang, P. Agarwal, and G. Wang, "On some new integral inequalities of Gronwall-Bellman-BIHari type with delay for discontinuous functions and their applications," Indagationes Mathematicae, vol. 27, no. 1, pp. 1-10, 2016.

[28] M. T. Malinowski, "Random fuzzy fractional integral equations-theoretical foundations," Fuzzy Sets and Systems, vol. 265, pp. 39-62, 2015.

[29] S. Salahshour, A. Ahmadian, N. Senu, D. Baleanu, and P. Agarwal, "On analytical solutions of the fractional differential equation with uncertainty: application to the Basset problem," Entropy, vol. 17, no. 2, pp. 885-902, 2015. 
[30] J. Tariboon, S. K. Ntouyas, and P. Agarwal, "New concepts of fractional quantum calculus and applications to impulsive fractional q-difference equations," Advances in Difference Equations, vol. 2015, article 18, 2015.

[31] X. Zhang, P. Agarwal, Z. Liu, and H. Peng, “The general solution for impulsive differential equations with Riemann-Liouville fractional-order $q \in(1,2)$," Open Mathematics, vol. 13, no. 1, pp. 908-930, 2015.

[32] T. Allahviranloo and M. B. Ahmadi, "Fuzzy Laplace transforms," Soft Computing, vol. 14, no. 3, pp. 235-243, 2010.

[33] E. ElJaoui, S. Melliani, and L. S. Chadli, "Solving second-order fuzzy differential equations by the fuzzy Laplace transform method," Advances in Difference Equations, vol. 2015, article 66, pp. 1-14, 2015.

[34] A. Jafarian, A. K. Golmankhaneh, and D. Baleanu, "On fuzzy fractional Laplace transformation," Advances in Mathematical Physics, vol. 2014, Article ID 295432, 9 pages, 2014.

[35] S. Arshad and V. Lupulescu, "Fractional differential equation with the fuzzy initial condition," Electronic Journal of Differential Equations, No. 34, 8 pages, 2011.

[36] S. Arshad and V. Lupulescu, "On the fractional differential equations with uncertainty," Nonlinear Analysis: Theory, Methods \& Applications, vol. 74, no. 11, pp. 3685-3693, 2011.

[37] N. V. Hoa and N. D. Phu, "Fuzzy functional integro-differential equations under generalized H-differentiability," Journal of Intelligent \& Fuzzy Systems, vol. 26, no. 4, pp. 2073-2085, 2014.

[38] S. Salahshour, T. Allahviranloo, and S. Abbasbandy, "Solving fuzzy fractional differential equations by fuzzy Laplace transforms," Communications in Nonlinear Science and Numerical Simulation, vol. 17, no. 3, pp. 1372-1381, 2012.

[39] T. Allahviranloo, S. Abbasbandy, and S. Salahshour, "Fuzzy fractional differential equations with nagumo and krasnoselskiikrein condition," in Proceedings of the 7th Conference of the European Society for Fuzzy Logic and Technology (EUSFLAT '11) and les Rencontres Francophones sur la Logique Floue et ses Applications (LFA '11), vol. 1, pp. 1038-1044, Aix-les-Bains, France, July 2011.

[40] H.-J. Zimmermann, Fuzzy Set Theory-And Its Applications, Kluwer Academic Publishers, Boston, Mass, USA, 2nd edition, 1992, With a foreword by L. A. Zadeh.

[41] T. Allahviranloo, S. Abbasbandy, M. R. Balooch Shahryari, S. Salahshour, and D. Baleanu, "On solutions of linear fractional differential equations with uncertainty," Abstract and Applied Analysis, vol. 2013, Article ID 178378, 13 pages, 2013. 

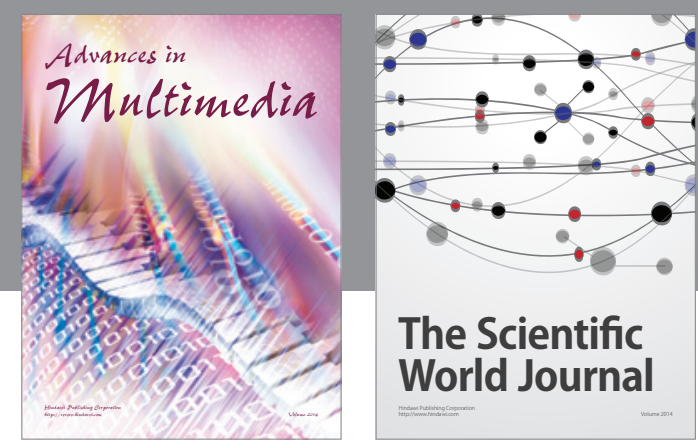

The Scientific World Journal
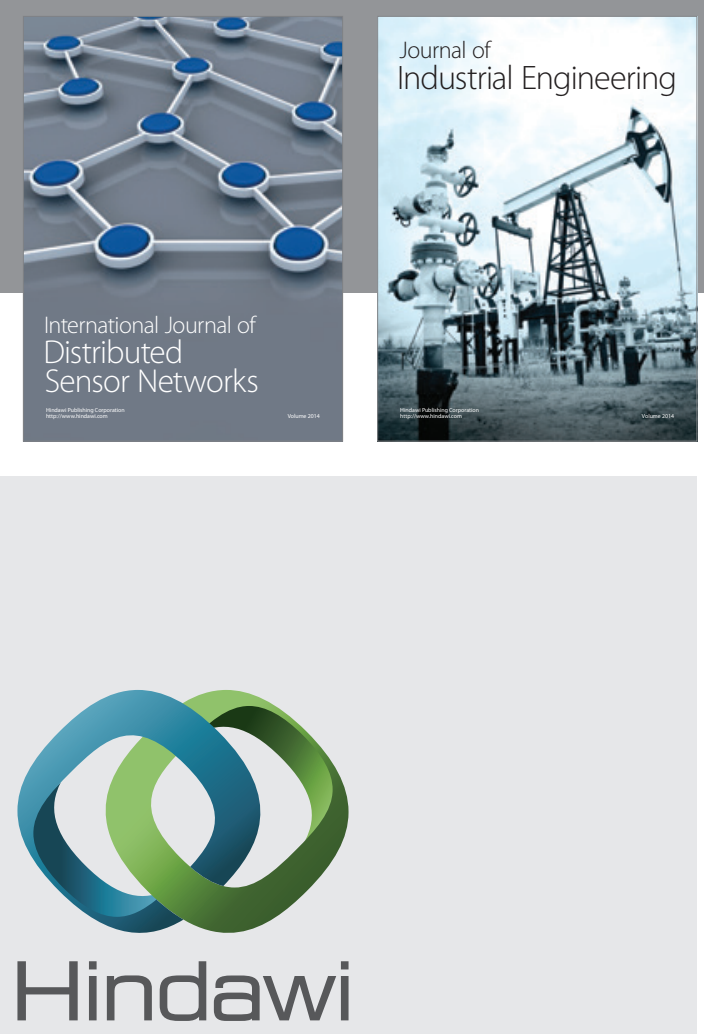

Submit your manuscripts at

http://www.hindawi.com

\section{Computer Networks} and Communications
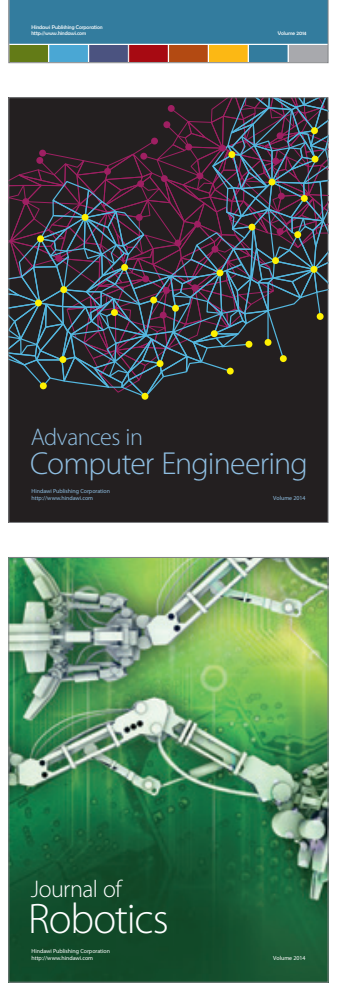
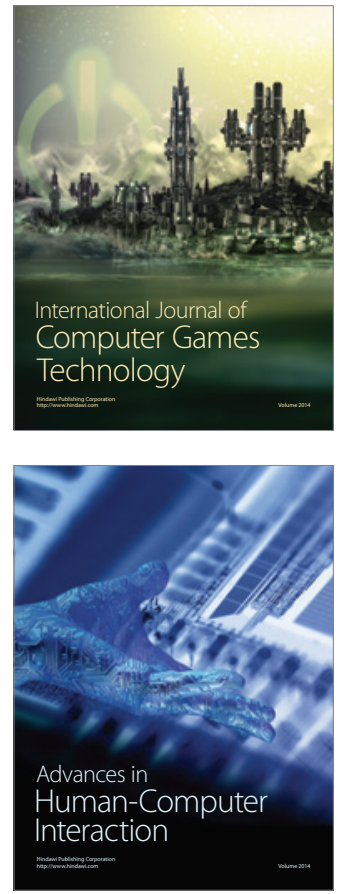
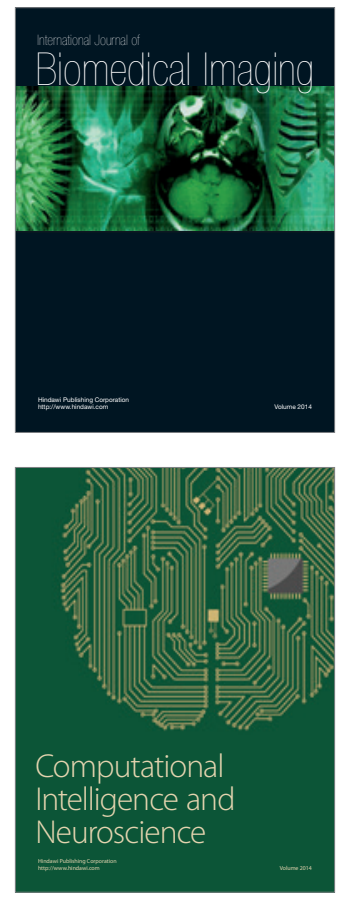
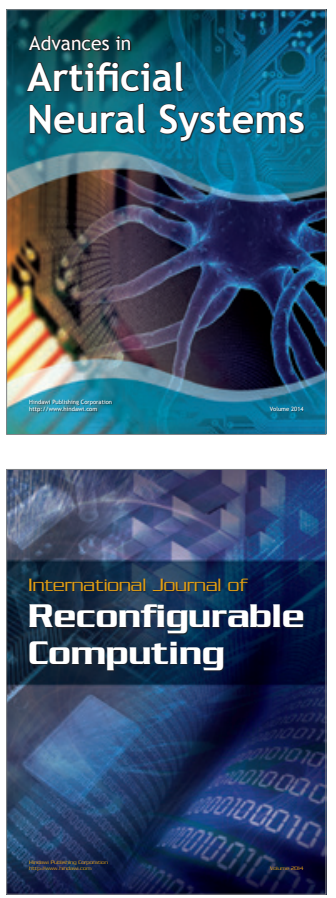
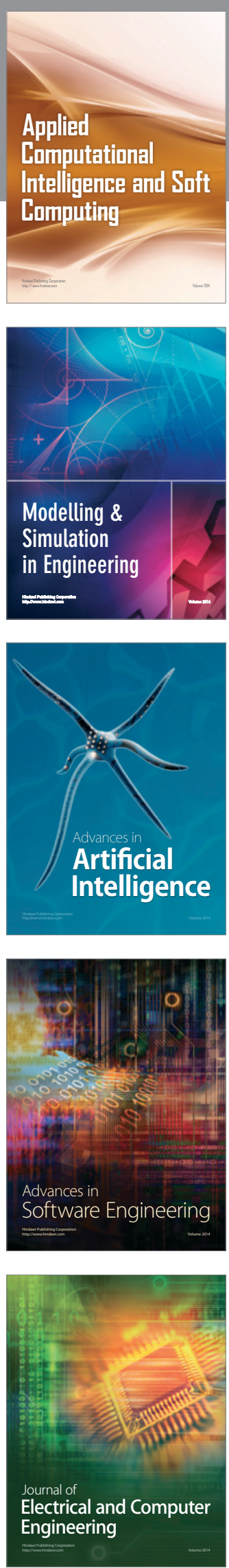